\title{
ASPECTS OF THE DETERMINATION OF EQUILIBRATION RATES BY NMR SPECTROSCOPY
}

\author{
John D. Roberts \\ Crellin Laboratory, California Institute of Technology, Pasadena, California \\ $91125, \mathrm{USA}^{\mathrm{a}}$
}

\begin{abstract}
The origins of the line-shape method for determining the rates of chemical and conformational equilibration by NMR spectroscopy are reviewed for the purpose of illustrating the factors which should be considered in obtaining accurate rate data by this very useful technique. Besides a number of illustrations of how changes in rates of conformational equilibration change NMR spectra taken from the literature, some new results using a simple and direct procedure for measurement of $\mathrm{NH}$ exchange rates of amides and hydrazines by naturalabundance ${ }^{15} \mathrm{~N}$ NMR will be presented.
\end{abstract}

\section{INTRODUCTION}

Without question, nuclear magnetic resonance (NMR) spectroscopy has greatly deepened our knowledge of chemical compounds and their reactions. No small part of this has been the application of NMR to the study of rate processes, either directly by following the rate of change of signal intensities with time or indirectly of equilibration rates by analysis of line shapes. The latter procedure will be the subject of this paper.

\section{DEVELOPMENT OF THE LINE-SHAPE PROCEDURE FOR MEASURING EQUILI- BRATION RATES BY NMR}

In this era of hectic change in both science and our daily lives, there is now a trend (or fad) for establishing the basis for how we got to where we are. "Searching for our roots" is the popular phrase. The taproot of NMR is of course the demonstration by Purcell ${ }^{2}$ and Bloch ${ }^{3}$ in 1946 that NMR signals could be obtained of protons in liquids and solids. This work of Bloch and Purcell was done with magnets of low homogeneity and the discovery of the chemical shift in 1950,4 and of spin-spin splitting in $1951^{5}$ required substantially greater resolution.

The first rootlet hinting at exchange processes was the discovery in $1951,{ }^{6}$ that the chemical shift of the $\mathrm{OH}$ proton of ethanol showed a temperature dependence, and this was almost immediately interpreted correctly by Liddel and Ramsey ${ }^{7}$ as arising from changes in association equilibria with temperature. "Since this association is by a hydrogen bond involving the hydrogen in the $\mathrm{OH}$ group, this hydrogen should experience a different magnetic shielding in the associated and unassociated state. If the correlation time for the lifetimes of the different states is sufficiently small (less than a millisecond), the hydrogen resonance will be observed at the frequency corresponding to the average shielding for the states." This rootlet was greatly nourished by the work of Gutowsky and Saika ${ }^{8}$ who showed that separate $\mathrm{OH}$ resonances for water and ethanoic acid could not be observed in mixtures of these substances but instead there was a common $O H$ resonance whose position was a linear function of the mole fractions of the components. Although Gutowsky and Saika ${ }^{8}$ did not provide any experimental examples of fast, intermediate, and slow exchange, they did use the Bloch equations to show theoretically how the line shapes would change with different preexchange lifetimes.

Apparently, the first published report of averaging of both shifts and spin-spin couplings came from Weinberg and Zimmerman' who showed that when water was added to ethanol, separate $\mathrm{OH}$ resonances could be obtained until the concentration of water reached about

Note a. Contribution No. 5863 from the Gates and Crellin Laboratories of Chemistry. 
$20 \%$ by weight. Above that concentration a single average line was obtained. Changes in the spin-spin coupling pattern of the $\mathrm{CH}_{2}$ were also observed over the concentration range where the lines coalesced. Curiously, there is no reference to Gutowsky and Saika or to Weinberg and Zimmerman in a later, otherwise superb, paper in 1956 by Arnold ${ }^{10}$ who constructed a permanent magnet, remarkable for the time, with which proton spectra could be taken at $30 \mathrm{MHz}$ having a resolution of about $0.5 \mathrm{~Hz}$. This magnet permitted Arnold to discover the second-order splitting between the $\mathrm{CH}_{2}$ and $\mathrm{CH}_{3}$ groups of ethanol, as well as produce excellent spectra of the collapse of the $\mathrm{OH}$ triplet of non-exchanging ethanol when made more than $5 \times 10^{-6} \mathrm{M}$ in $\mathrm{HCl}$. Arnold calculated line shapes for the $\mathrm{OH}$ proton of ethanol as a function of the exchange rate, $\tau$, and showed how these could be correlated with the observed line shapes. The difficulty of getting reproducible line shapes as a function of $\mathrm{HCl}$ concentration prevented this paper from being the definitive initial example of the power of the NMR method for measuring equilibration rates.

In 1955, Phillips ${ }^{11}$ demonstrated that rotation about the $\mathrm{C}-\mathrm{N}$ bond of $\underline{N}, \underline{N}$-dimethylmethanamide was slow and in 1956 Gutowsky and Holm 12 showed how the gradual averaging of the chemical shifts of the methyl groups with increasing temperature could be used to obtain an activation energy for the bond rotation. This could have been the definitive initial paper on determination of rates from line shapes but, in fact, it gave NMR measurements of rates a very bad aroma for several years. The problem was that Gutowsky and Holm related the rates to line separations and got an activation energy for rotation of $\mathrm{N}, \mathrm{N}$-dimethylmethanamide of $7 \pm 3 \mathrm{kcal}$ which for a process for which having $\Delta \underline{G} \neq$ of about $\overline{19} \mathrm{kcal}$ means that $\Delta S \ddagger$ has to be -32 eu which seems quite unreasonably large. Subsequent measurements of activation energies of rotation about amide bonds by many researchers gave a wide disparity of values ranging up to $25 \mathrm{kcal}$. Two difficulties were common. One was that temperatures of NMR samples are not easy to control precisely or easy to measure. The other was the Gutowsky-Holm treatment, which requires substantial correction whenever the chemical-shift difference changes with temperature. If the chemical-shift difference decreases with temperature, as it does with $\mathrm{N}, \mathrm{N}$-dimethylmethanamide, before chemicalshift averaging really becomes important, and line separations are used as the criterion of averaging, the rate will appear to change over a wider temperature range than it should. This means that the activation energy will appear to be smaller than its actual value. The theoretical spectra shown in Figure 1, calculated by Gutowsky and Holm's equation show

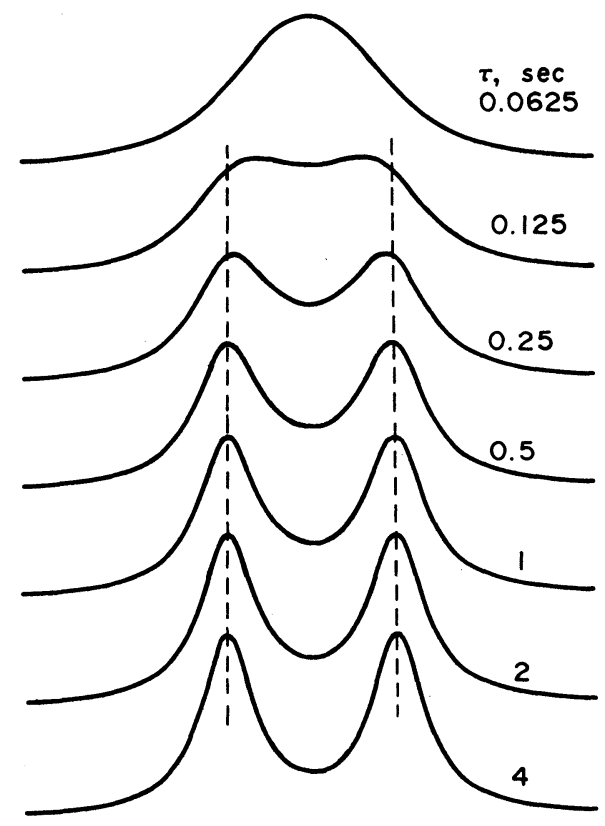

Figure 1. Line shapes calculated as a function of $\tau$ for two-site exchange by equations of Gutowsky and Holm 12 with $\delta \omega=6 \mathrm{~Hz}, \mathrm{~T}_{2}=0.9 \mathrm{sec}$, showing how there is little movement of the peaks toward one another until near the coalescence point. 
how the line separations do not in fact change very much with increasing rate until the sample gets close to the coalescence point. The coalescence point is of course easy to measure and, as a result, there is usually good agreement between different investigators as to the free energy of activation--whether or not they agree on the enthalpy of activation by a factor of two.

The enthalpy of activation can be determined reasonably accurately by measuring the coalescence temperature for the same sample in spectrometers with different magnetic fields and thus a range of chemical-shift differences. The relative rate at the coalesence point is proportional to the chemical shift so that between $30 \mathrm{MHz}$ and $600 \mathrm{MHz}$ one could cover a rate range of a factor of 20 rather accurately.

Subsequent to the work on rotational barriers of amides came the discovery that barriers to rotation could be determined for $\mathrm{C}-\mathrm{C}$ single bonds 13,14 and of ring inversion in cyclohexane. 15 The floodgates for study of conformational equilibration were then open and, because of the complexity of the spectra which began to be observed, better procedures for calculating line-shape changes as a function of rate became necessary.

Before proceeding to these, it may be well to consider why the signals are averaged and it is interesting that both the Bloch equations which are regarded as essentially classical equations and the uncertainty principle predict that line broadening, coalescence, and then line narrowing will occur as the exchange rate increases. The uncertainty principle suggests that, if the lifetime of a state is short, there will be an uncertainty in the energy of the state and line broadening will occur. Line narrowing above the coalescence point is a consequence of the fact that the uncertainty in the energy of the averaged state becomes less. The Bloch equations predict line broadening by inclusion of the $T_{1}$ and $T_{2}$ relaxation times as well as the exchange rates and these rates essentially amount to uncertainty in the lifetimes of the states.

A more precise quantum mechanical approach to the problem of calculating line shapes was introduced by Sack 16 and Kaplan 17 using the density-matrix procedure. This was a great advance, because it permitted introduction of spin-spin coupling for situations in which the chemical shifts and couplings are of comparable magnitude (AB vs. AX in NMR parlance). The Kaplan procedure was soon programmed to calculate line shapes for AB-type systems ${ }^{18}$ and many new interesting applications became available. Still later Binsch ${ }^{19}$ used the Liouville representations to produce much more efficient algorithms for computing line shapes and these can be used with very complex spin systems, which as we will see offer substantial advantage for measuring larger changes in rate for a particular variety of exchange.

The line-shape procedure as described here is only one of several methods for determining rates of equilibration by NMR. Pulse methods such as the spin-echo technique 20 are very powerful as is also double resonance. 21 Because the interpretation of spectra produced by these procedures is complex, they have not been used much by organic chemists and consequently they will not be discussed further here.

One might well wonder whether equilibration rates for a reaction $A \rightleftarrows B$ measured by such an esoteric procedure as looking at line broadening in NMR spectra are really the same rates which one might determine by optical rotation, titration and the like, or even by following the change of signal intensity in an NMR tube when pure A goes to equilibrium with B. This point has been investigated and the differences in rate are not larger than the experimental precision of the measurements. 22

\section{SOME PRACTICAL EXAMPLES OF THE LINE-SHAPE PROCEDURE}

In many cases, the line-shape method is too difficult or impossible to apply because the NMR spectra are just too complicated to be calculated even without the extra complications produced by exchange. An example is cyclohexane below the temperature where the signals of the axial and equatorial hydrogens are averaged to a single line. One technique which has been used with great success by Anet 23 is to use massive deuteration so that there is only a single proton left in the molecule. The line-shape problem for cyclohexane- $d_{11}\left(w_{i t h}\right.$ deuterium decoupling) then reduces to the one presented by a simple two-site, no spin-spin coupling system discussed earlier. Another approach is to use fluorine-labeling 24 because fluorine has large chemical-shift differences and useful information can be obtained over 


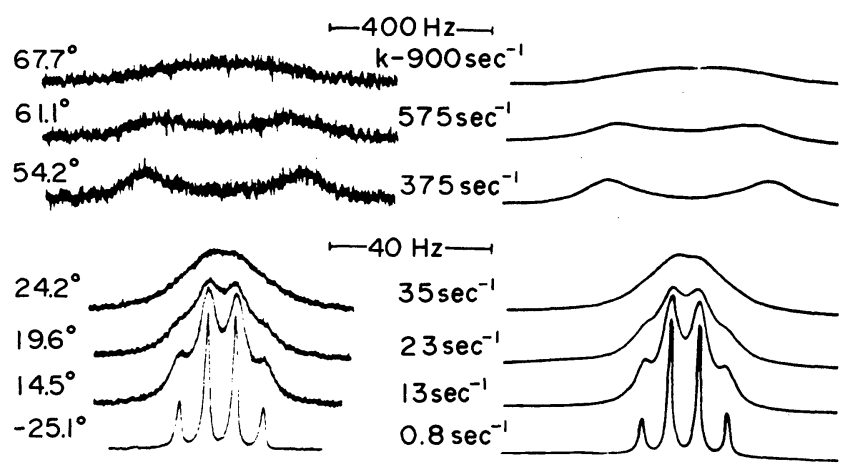

Figure 2. Comparison of experimental $19 \mathrm{~F}$ spectra of 1 as a function of temperature, below and at the main coalescence point, with calculated spectra as a function of $\mathrm{k}(=1 / \tau)$ in $\mathrm{sec}^{-1}, \mathrm{X}=-\mathrm{F}$.

both a wider temperature range and at lower temperatures by observing fluorine than is possible by observing protons. The problem with fluorine labeling is that if one is really interested in activation parameters for the unlabeled compound, it is hard to be sure that the fluorines are not themselves causing unusual effects. 25

In general, massive deuteration is not desirable if it can be avoided. The reason is that spectral complexities are extremely useful in extending the range of temperatures over which rates can be measured by the NMR procedure. An excellent example is provided by the (1-(trifluoromethyl)-2, 2, 2-trifluoroethylidene)-4-substituted benzenamines (1) which undergo rather rapid degenerate geometrical isomerization at room temperature (Figure 2). 26<smiles>[X]c1ccc(N=C(C(F)(F)F)C(F)(F)F)cc1</smiles>
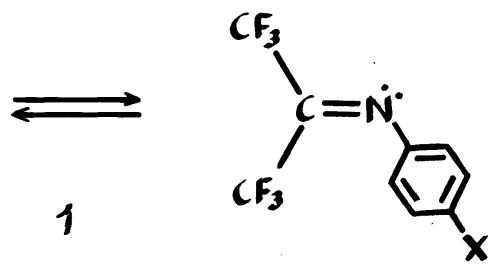

The chemical-shift differences between the fluorines of the trifluoromethyl groups of 1 when isomerization is slow are large and there is, in addition, the expected quartets from longrange spin splittings involving the non-equivalent fluorines. These couplings are washed out over about a $40^{\circ}$ change in temperature and an additional $40^{\circ}$ is required to reach coalescence. Measurements of the rate above the coalescence point can only be made by measuring the line width of the single averaged fluorine resonance of the trifluoromethyl groups, and while this is reasonably accurate it does not compare with fitting the subtle line-shape changes of the quartet in the slower exchange region.

A current study 27 of the corresponding difluoromethyl derivatives 2 shows that these substances have substantial NMR advantages over 1 , because of the extra complications introduced in the spectra by the ${ }^{1} \mathrm{H}-{ }^{19} \mathrm{~F}$ spin-spin splittings (Figure 3 ). Comparison of Figures
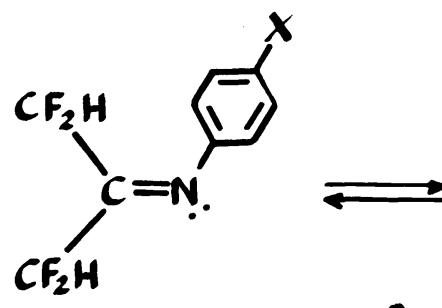

2

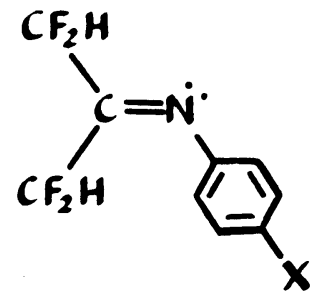




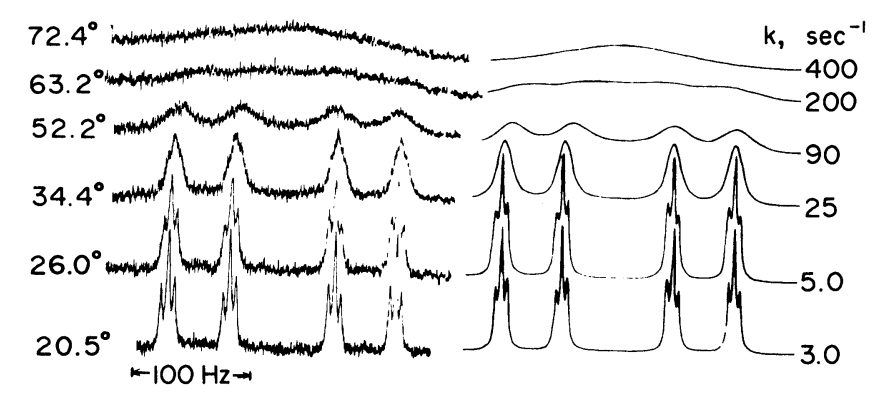

Figure 3. Comparison of experimental ${ }^{19} \mathrm{~F}$ spectra of 2 as a function of temperature, below and at the main coalescence point, with calculated spectra as a function of $\mathrm{k}(=1 / \mathrm{T})$ in $\sec ^{-1}, \mathrm{X}=-\mathrm{NO}_{2}$.

2 and 3 shows that, when isomerization is slow, the ${ }^{19} \mathrm{~F}$ spectrum of 2 is a quartet of triplets and we can follow the collapse of first the triplets and then the quartets to the coalescence point. Above the coalescence point (Figure 4), the spectrum gradually splits into a doublet of triplets as expected for an $\mathrm{A}_{2} \mathrm{~A}_{2}^{\prime} \mathrm{XX}^{\prime}$ arrangement in the fast-exchange limit. This greatly extends the range over which the rate can be measured reasonably accurately. The total range encompassed by the changes in line shape covers a range of rates in the order of about 25, 000 which is orders of magnitude larger than possible by measuring coalescence points at different magnetic fields or line separations.

The epitome of line-shape analysis has been recently provided by Binsch ${ }^{28}$ for the $\mathrm{ABC} \rightarrow \mathrm{DEF}$ system of the ring protons of $\mathrm{N}$-trideuteriomethyl-2-cyanoazacyclopropane (3) which undergoes both slow to fast (nondegenerate) inversion at nitrogen and a change of the equilibrium constant $\mathrm{K}$ with increasing temperature. The extraordinary changes of the proton spectrum with temperature and the way that these can be duplicated by theoretical calculations are a joy to behold.<smiles>N#CC1C2[C@H](C(=O)[O-])[C@@H]12</smiles>

$K \neq 1$

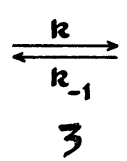

3

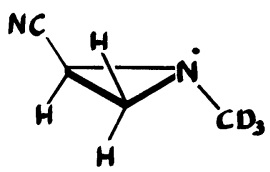

Natural-abundance ${ }^{13} \mathrm{C}$ NMR spectra have been widely used for the study of equilibration rates since the first determination of activation parameters for inversion of $1,1,3,3-$ tetramethylcyclohexane in 1970.29 An excellent review on this subject was published in 1977 which gives activation parameters for more than 180 different compounds. 30 With most organic compounds, proton-coupled ${ }_{13} \mathrm{C}$ spectra will be too çomplex to be useful. Because of the low natural-abundance of ${ }^{13} \mathrm{C}$, proton-decoupled ${ }^{13} \mathrm{C}$ spectra of exchanging

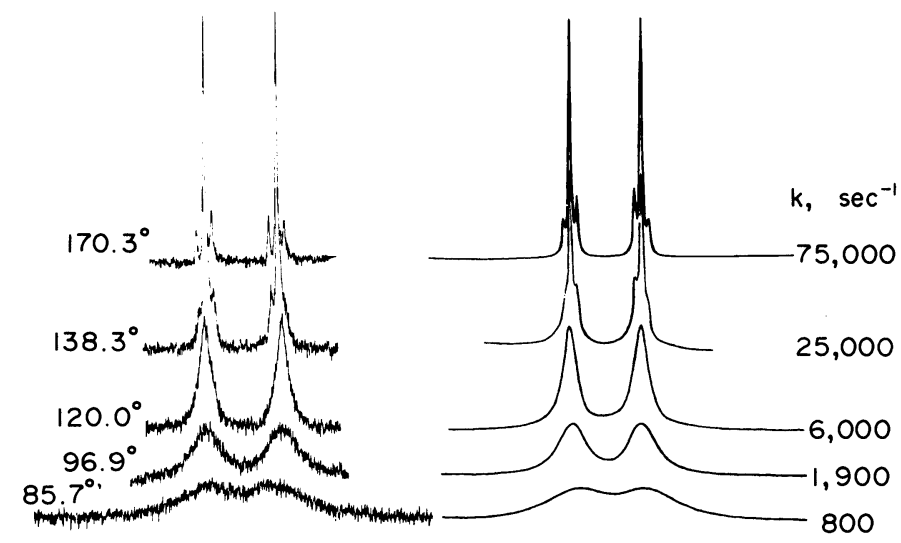

Figure 4. Same as Figure 3 but above the coalescence point. 
systems only involve averaging of chemical shifts unless some magnetic nucleus such as fluorine or phosphorus is present in the molecule. Proton-decoupled ${ }^{13} \mathrm{C}$ spectra lack the fine structure which helps in extracting rates from changes in line shapes as discussed above and, if the chemical shifts are large, it may be difficult to have a large enough signal-to-noise ratio in the coalescence region to obtain accurate rate data. Supplementation of the line-shape procedure by the double-resonance spin-saturation procedure 21 appears to be very useful with ${ }^{13} \mathrm{C}$ spectra. ${ }^{30}$

Nitrogen-15 NMR has so far had relatively limited use in rate studies. At the naturalabundance level, the sensitivity of detection is about $6 \times 10^{11}$ more difficult than protons at the same equivalent concentration and this means that either rather large samples or enrichment in the isotope are necessary. Serious problems with ${ }^{15} \mathrm{~N}$ NMR are the great variability of the relaxation times of the nucleus, the rather extreme sensitivity of the resonances of many kinds of nitrogen to paramagnetic ions, and the variability of the nuclear Overhauser enhancement (negative) produced by proton decoupling.

An illustrative example of ${ }^{15} \mathrm{~N}$ chemical-shift averaging has been published for mesotetraphenylporphyrin 4. ${ }^{31}$ A number of attempts to obtain the natural-abundance ${ }^{15} \mathrm{~N}$

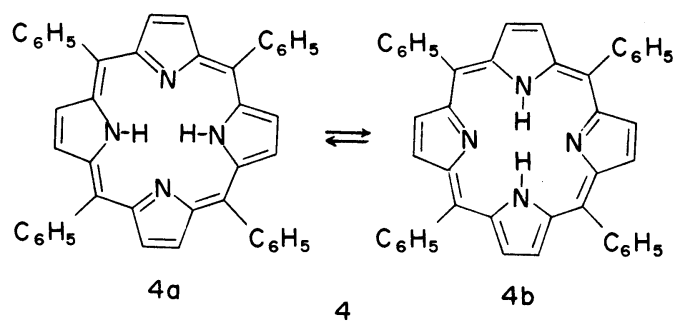

spectrum of this substance were unsuccessful under conditions which were known to be satisfactory for related structures. The compound was then prepared enriched to the $95 \%$ level in ${ }^{15} \mathrm{~N}$ and, when the spectrum of the product was taken, it was found to give a single resonance with a line width of about $180 \mathrm{~Hz}$ at $18.25 \mathrm{MHz}$. This suggested an exchange process such as an equilibrium between $4 \mathrm{a}$ and $4 \mathrm{~b}$ and, when the sample was cooled, the broad singlet became a doublet with a chêmical-shift difference of 109 ppm (Figure 5). The coalescence temperature for 4 of about $15^{\circ} \mathrm{C}$ suggests that $\Delta \mathrm{G}^{\ddagger}$ is about $11 \mathrm{kcal}$ in reasonable agreement with $\Delta \underline{G}^{\ddagger}$ obtaîned from the changes of the proton spectrum of 4 with temperature. 32

Similar exchange broadening ocaurs at room temperature when 4 is dissolved in 2-propanone containing less than the equivalent of trifluoroethanoic acid to form its dication 5 . No ${ }^{15} \mathrm{~N}$ resonances were detected at room temperature because of being close to the coales cence point for equilibration of $4 \mathrm{a}$ with $\underset{\sim b}{\mathrm{~b}}$ and both with 5 .

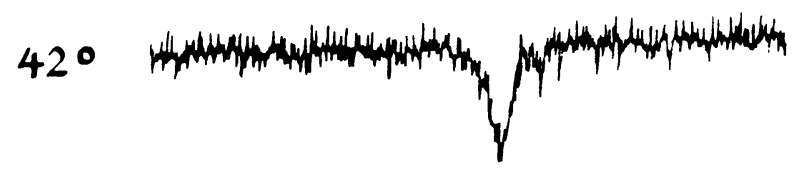

$10^{\circ}$
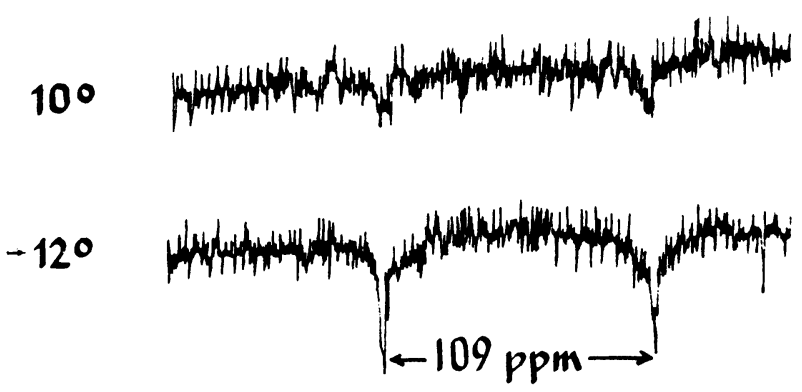

Figure 5. Changes in the proton-decoupled ${ }^{15} \mathrm{~N}$ spectrum of 4 at $18.25 \mathrm{MHz}$ with temperature in trichloromethane solution. 


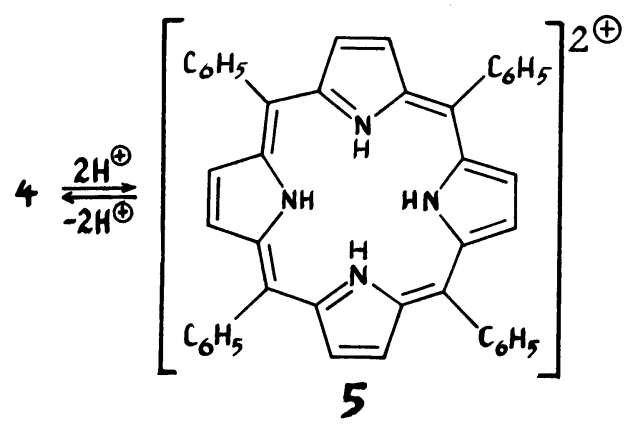

The ${ }^{15} \mathrm{~N}$ spectra of Figure 6 show that, at $-10^{\circ} \mathrm{C}$, the equilibrium between 4 and 5 is slow and 5 gives a sharp narrow line because all of its nitrogens are equivalent. Thê lines of 4 are broad because the $4 \mathrm{a} \rightleftarrows 4 \mathrm{~b}$ equilibration is not completely frozen out at $-10^{\circ}$. At $38^{\circ}$, $4 \mathrm{a} \approx 4 \mathrm{~b}$ is above the coâlescence temperature for the nitrogen resonances of 4 and exchange with $\widetilde{5}$ produces substantial line broadening. This is an interesting set of rates because exchânge between the imidazole tautomers and between imidazole and imidazolium ion is very fast under comparable conditions. 33
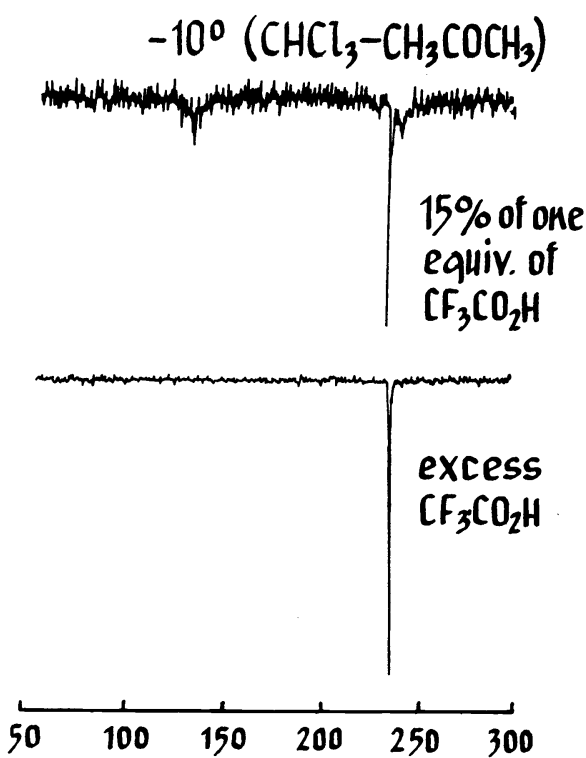

Figure 6. The ${ }^{15} \mathrm{~N}$ spectra of 4 in a 2-propanone - trichloromethane mixture at $-10^{\circ} \mathrm{C}$ containing trîfluoroethanoic acid.

Nitrogen-15 NMR has recently shown to be very useful at the natural-abundance level to measure acid- or base-catalyzed competitive exchange proton rates of hydrazines, guanidines and amides. Exchange between the hydrogens of phenyldiazane is one example. 34 The gated proton-coupled ${ }^{15} \mathrm{~N}$ spectra of neat phenyldiazane in Figure 7 show ${ }^{15} \mathrm{~N}-\mathrm{H}$ couplings that indicate at $20^{\circ} \mathrm{C}$ exchange of the - $\mathrm{NH}$-proton must be quite slow because of the sharpness of the $\mathrm{Nl}$ doublet. On the other hand, exchange of the $-\mathrm{NH}_{2}$ protons is fast enough to produce significant line broadening of the $\mathrm{N} 2$ triplet resonance. At $60^{\circ} \mathrm{C}$, slight exchange broadening of the $\mathrm{Nl}$ doublet is evident but the triplet resonance of $\mathrm{Nl}$ has degenerated to a broad singlet. The pattern of these exchange rates is expected on the basis of the low basicity of N1 compared to N2. Surprisingly, the exchange rates of the protons attached to $\mathrm{Nl}$ and $\mathrm{N} 2$ are roughly the same in methylsulfinylmethane, ethanol and ethanolwater (Figure 8). Less surprising is the increase in exchange rate in the more acidic solvents trifluoroethanol and trifluoroethanoic acid. 


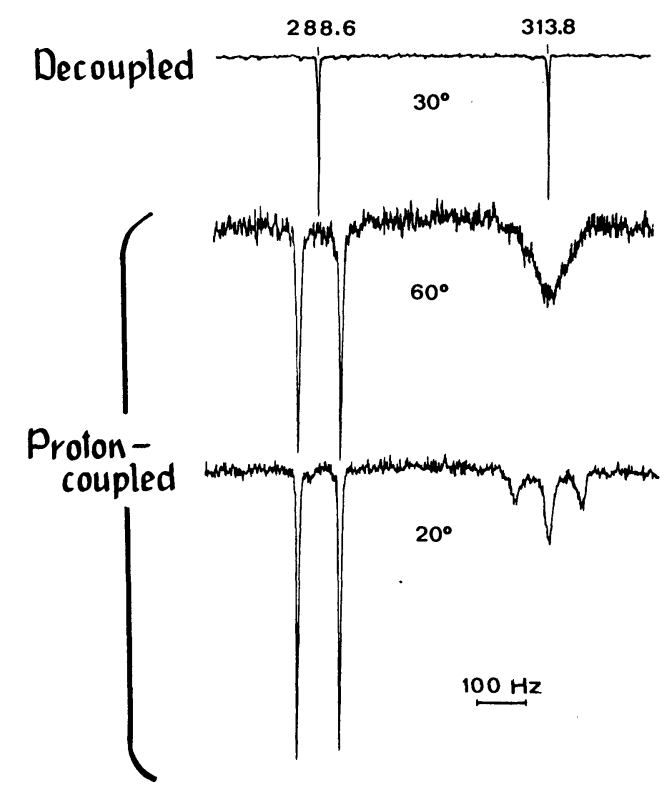

Figure 7. Natural-abundance ${ }^{15} \mathrm{~N}$ spectrum of neat phenyldiazane as a function of temperature and proton decoupling. The doublet on the left in the proton-coupled spectrum arises from the $-\mathrm{NH}$-nitrogen.

Recently, ab initio calculations have been reported ${ }^{35}$ which suggest that the $\mathrm{NH}_{2}$ nitrogens of the carbamimidoylamine group of the conjugate acid of arginine (6) should be substantially more positive than the $\mathrm{NH}$ nitrogen.<smiles>N=[14C](N)NCCC(N)C(=O)O</smiles>

This would suggest that the protons on the $-\mathrm{NH}_{2}$ groups should undergo base-catalyzed exchange much faster than that on the - NH- group. The changes in the proton-coupled ${ }^{15} \mathrm{~N}$ spectra of 6 in water as a function of $\mathrm{pH}$ (Figure 9) indicate that this expectation is not realized. The doublet of the $-\mathrm{NH}$-nitrogen and the triplet of the $-\mathrm{NH}_{2}$ nitrogens broaden almost equally rapidly. ${ }^{36}$ Thus, if one allows for a statistical factor of two favoring $-\mathrm{NH}_{2}$ over $-\mathrm{NH}$, it is clear that the $-\mathrm{NH}$-protons exchange more rapidly than the $-\mathrm{NH}_{2}$ protons.

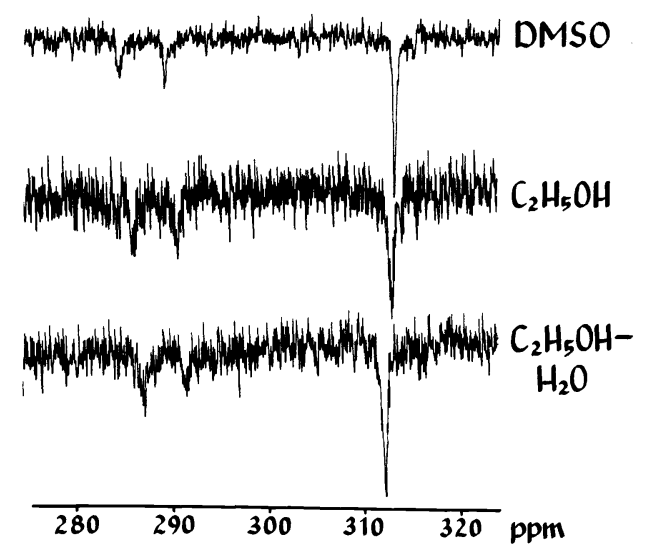

Figure 8. Natural-abundance ${ }^{15} \mathrm{~N}$ spectra of $20 \% \mathrm{v} / \mathrm{v}$ phenyldiazane in various solvents at $30^{\circ} \mathrm{C}$, protons coupled. 


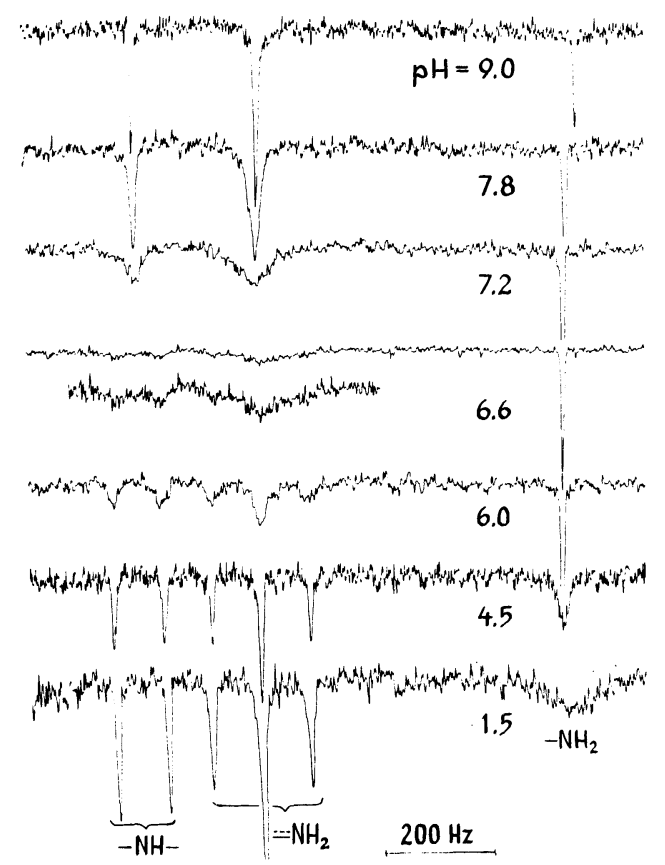

Figure 9. Natural-abundance ${ }^{15} \mathrm{~N}$ spectra of $1.5 \mathrm{M}$ arginine in water as a function of $\mathrm{pH}$, protons coupled.

This same technique has been used to study competitive exchange rates of $\mathrm{NH}$ protons of ureas and thioureas, ${ }^{37}$ lactams 38,39 and thiolactams, ${ }^{39}$ and amides. 37,40 The exchange rates of the $\mathrm{NH}_{2}$ protons of primary amides are hard to measure with proton spectra because of ${ }^{14} \mathrm{~N}$ quadrupole relaxation, which usually occurs at just the right rate to greatly broaden the $\mathrm{NH}$ proton resonances. The same thing occurs with secondary amides, such as $\mathrm{RCONHCH}$, but with these the spin-spin splitting of $-\mathrm{CH}_{3}$ proton resonances by the $\mathrm{NH}$ proton can be used as a probe of exchange rates. 41

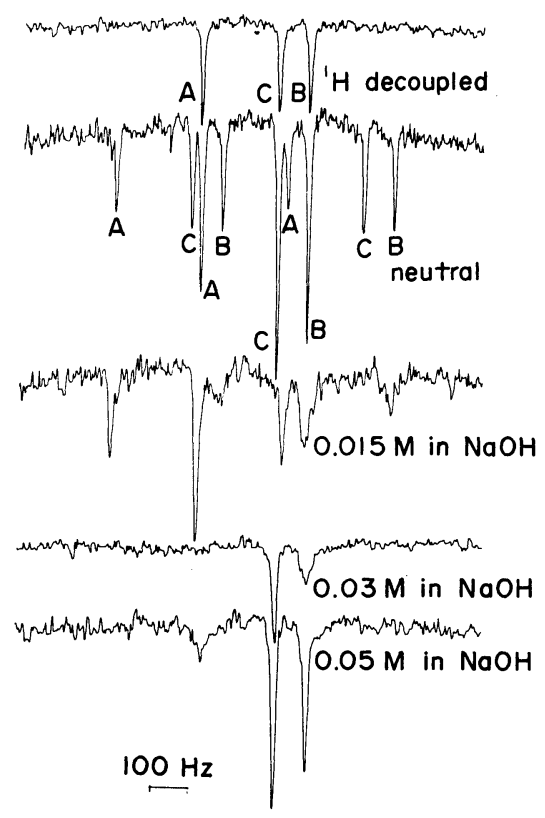

Figure 10. Natural-abundance ${ }^{15} \mathrm{~N}$ spectra of a mixture of butanamide (A), benzenecarboxamide (B), and nicotinamide (C) in methylsulfinylmethane, either neutral or with added sodium hydroxide. 


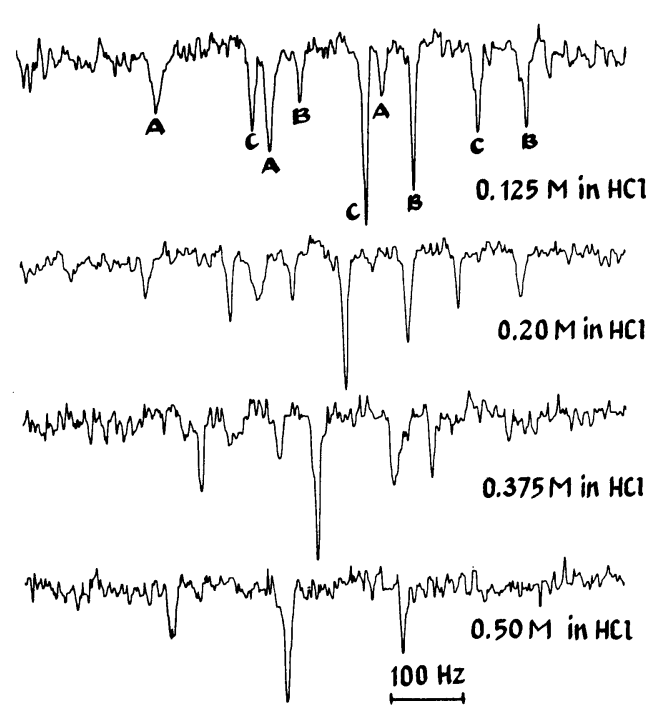

Figure 11. Same as Figure 10 except that hydrochloric acid added rather than sodium hydroxide.

The power of ${ }^{15} \mathrm{~N}$ NMR to measure relative exchange rates for primary amides is illustrated by Figures 10 and 11 which show spectra of solutions containing butanamide, benzenecarboxamide and 3-azabenzenecarboxamide (nicotinamide) in methylsulfinylmethane solution as a function of different amounts of added acid and base. 40 With added base, the rate ratios are approximately 3-azabenzenecarboxamide:benzenecarboxamide: butanamide as 100:10:1 while, in acid, the ratio is about 1:1000:5000. In acid solution, the ring nitrogen of the 3-azacarboxamide becomes protonated and makes the $-\mathrm{NH}_{2}$ nitrogen less able to accept a proton than the other amides. In basic solution, the superior electron-attracting powers of the 3-azaphenyl and phenyl groups assist the removal of the $-\mathrm{NH}_{2}$ protons.

\section{REFERENCES}

1. Supported by the National Science Foundation and the Public Health Service, Research Grant No. GM-11072 from the Division of General Medical Sciences.

2. E. M. Purcell, E. C. Torrey, and R. V. Pound, Phys. Rev. , 69, 37 (1946).

3. F. Bloch, W. W. Hansen, and M. E. Packard, Phys, Rev., 69, 127 (1946).

4. W. G. Proctor and F. C. Yu, Phys. Rev., 77, 717 (1950); W. C. Dickinson, Phys. Rev., 77, 736 (1950).

5. H. S. Gutowsky and C. J. Hoffman, Phys. Rev., 80, 110 (1950); E. L. Hahn, Phys. Rev., 80, 550 (1950).

6. J. T. Arnold and M. E. Packard, J. Chem. Phys., 19, 1608 (1951).

7. U. Liddel and N. F. Ramsey, J. Chem. Phys., 19, 1608 (1951).

8. H. S. Gutowsky and A. Saika, J. Chem. Phys., 21 , 1688 (1953); see also H. S. Gutowsky, D. W. McCall, and C. P. Slichter, J. Chem. Phys., 21, 279 (1953).

9. I. Weinberg and J. R. Zimmerman, J. Chem. Phys., 23, 748 (1955).

10. J. T. Arnold, Phys. Rev., 102, $136(1956)$.

11. W. D. Phillips, J. Chem. Phys., 23, 1363 (1955).

12. H. S. Gutowsky and C. H. Holm, J. Chem. Phys., 25, 1228 (1956).

13. P. M. Nair and J. D. Roberts, J. Am. Chem. Soc., 79, 4565 (1957).

14. W. D. Phillips, Ann. N. Y. Acad. Sci., 70, 817 (1958).

15. F. R. Jensen, D. S. Noyce, C. H. Sederholm, and A. J. Berlin, J. Am. Chem. Soc., 82, 1256 (1960).

16. R. A. Sack, Mol. Phys., 1, 163 (1958).

17. J. I. Kaplan, J. Chem. Phys., 28, 278 (1958); S. Alexander, J. Chem. Phys., 37, 967, 974 (1962); 38, 1787 (1963).

18. M. Saunders and F. Yamada, J. Am. Chem. Soc., 85, 1882 (1963); J. L. Beauchamp, Undergraduate Thesis, California Institute of $\mathrm{T}$ echnology, 1964. 
19. G. Binsch, J. Am. Chem. Soc., 91, 1304 (1969); see also G. Binsch, Chap. 3, L. M. Jackman and F. A. Cotton, Eds., Dynamic Nuclear Magnetic Resonance Spectroscopy, Academic Press, Inc., New York, (1975).

20. E. L. Hahn, Phys. Rev. , 80, 580 (1950); see L. W. Reeves, Chap. 4, L. M. Jackman and F. A. Cotton, Eds., Dynamic Nuclear Magnetic Resonance Spectroscopy, Academic Press, Inc., New York, 1975.

21. S. Forsén and R. A. Hoffman, J. Chem. Phys., 39, 2982 (1963).

22. An especially nice example was reported by M. Oki at the Gordon Conference on Stereochemistry, Plymouth, N. H. , July 19, 1978 wherein the rate of rotation about the $\mathrm{C} 9-\mathrm{Cl}^{\prime}$ bond of 9-(2-(1-methylethyl)phenyl) fluorene was measured by line-shape analysis of proton and carbon spectra and also by the change in proton signal intensity with time of a single rotational isomer. Excellent agreement was obtained for all of the methods. See also M. Nakamura, N. Nakamura, and M. Oki, Bull. Chem. Soc. Japan, 50, 2986 (1977).

23. F. A. L. Anet and A. J. R. Bourn, J. Amer. Chem. Soc., 89, 760 (1970).

24. J. D. Roberts, Chem. in Britain, 2, 529(1966) and references cited therein.

25. For one example, see R. D. Stolow, T. W. Giants, and J. D. Roberts, Tetrahedron Lett. , 55, 5777 (1968).

26. G. E. Hall, W. J. Middleton, and J. D. Roberts, J. Am. Chem. Soc., 93,4778 (1971).

27. R. J. Cook (Kalamazoo College) and V. L. Gray, unpublished research.

28. D. Hofner, I. Tamir, and G. Binsch, Org. Mag. Res., 11, 172 (1978).

29. D. Doddrell, C. Charrier, B. L. Hawkins, W. O. Crain and J. D. Roberts, Proc. Nat. Acad. Sci., U.S., 67, 1588 (1970).

30. B. E. Mann, Prog. in NMR Spect., 11, 95 (1977).

31. D. Gust and J. D. Roberts, J. Am. Chem. Soc., 99, 3637 (1971).

32. C. B. Storm and Y. Teklu, J. Am. Chem. Soc. , 94, 1745 (1972); C. B. Storm, Y. Teklu, and E. A. Sokolowski, Ann. N. Y. Acad. Sci., 206, 631 (1973); R. J. Abraham, G. E. Hawkes, and K. M. Smith, Tetrahedron Lett. , 71 (1974).

33. E. K. Ralph and E. Grunwald, J. Am. Chem. Soc., 91, 2422 (1969).

34. I. Yavari and J. D. Roberts, J. Am. Chem. Soc. , 100,4662 (1978).

35. D. A. Dixon and W. N. Lipscomb, J. Biol. Chem. , 251, 5992 (1976).

36. I. Yavari and J. D. Roberts, Biochem. Biophys. Res. Comm., 83, 635 (1978).

37. I. Yavari and J. D. Roberts, J. Phys. Chem., in press.

38. I. Yavari and J. D. Roberts, J. Am. Chem. Soc. , 100, 5217 (1978).

39. I. Yavari and J. D. Roberts, Tetrahedron Lett. $2 \overline{491}$ (1978).

40. I. Yavari and J. D. Roberts, Proc. Nat. Acad. Sci. , U. S., in press.

41. A. Berger, A. Loewenstein and S. Meiboom, J. Am. Chem. Soc., 81, 62 (1959). 